\begin{abstract}
We have developed a novel link layer architecture that provides multiple quality of service points simultaneously over wireless Internet links. Our approach enhances the performance of diverse applications over error-prone links. We discuss the performance problems of Internet protocols over wireless links, presenting as a case study our measurements on a wireless LAN, and argue that it is preferable to handle wireless impairments at the link layer. We present a simulation study of various link layer enhancements, and their impact on TCP and

UDP performance. O ur results show that different approaches are preferable for each type of application. We thus propose a

multiservice link layer approach that supports multiple link layer mechanisms over a single link. O ur scheme is transport-

protocol-independent and customizable for the underlying wireless link technology. While our approach can be directly deployed

on the existing Internet, it also provides support for future Q oS-aware protocols and applications. O ur simulation

results show that our approach uniformly improves the performance of both TCP and U DP applications.
\end{abstract}

\title{
Link Layer Support for Quality of Service on Wireless Internet Links
}

\author{
George Xyl omenos and George C. Polyzos, \\ Univer sity of California-San Diego
}

uring the past few years wireless communications have become very popular, with a multitude of competing technologies and service models available to the public. Cellular telephony (CT) systems are evolving worldwide to fully digital technologies, while wireless local area networks (W LANs) that transparently link wireless hosts to the Internet are becoming cheaper and more interoperable. These systems, despite their differences, share characteristics that set them apart from both traditional (geostationary) satellite and wired links: low propagation delays compared to satellites and high error rates compared to wired links. Their error behavior varies, sometimes rapidly, due to factors such as interference, multipath fading, atmospheric conditions, and possible user mobility, in a generally unpredictable manner. Due to economic and technological factors, typical wireless links are slower than wired ones, making them the most likely bottleneck of end-to-end paths.

The popularity of wireless systems has generated interest in their integration into the Internet. A Ithough superficially easy, given the minimal requirements of I nternet protocols, this task is complicated by hidden protocol dependencies on wired media. In addition, the unpredictable performance of wireless links hinders the evolution of the Internet to quality of service (QOS) provision. In the following section we discuss the causes and extent of these performance problems by reviewing previous work and presenting our own measurements. We argue that it is preferable to handle wireless errors at the link layer. We then present a simulation study of various link layer enhancement mechanisms, showing that different solutions are preferable for each type of application. We thus describe a novel multiservice link layer architecture that provides multiple simultaneous services over a single link to support diverse application requirements. We show how our approach can be deployed on the existing Internet or integrated with future Q oS-oriented protocols. Finally, we present simulation results showing that our architecture can uniformly improve the performance of multiple applications.

\section{Internet Protocol Performance over Wireless Links}

\section{The Impact of Wireless Impairments}

The Internet offers two main protocol choices at the transport layer. U D P does little more than provide direct access to IP, leaving higher-layer protocols or applications to deal with the limitations of IP's best effort datagram delivery service. TCP offers instead a reliable byte stream service to its users, also taking care of flow and congestion control. TCP receivers generate cumulative acknowledgments for data received in sequence, while TCP senders retransmit data when they receive multiple (usually three) duplicate acknowledgments. Since IP can reorder datagrams, fewer duplicate acknowledgments may not signify losses. TC P dynamically tracks the round-trip delay on the end-to-end path and times out when acknowledgments are not received in time, retransmitting unacknowledged data. TCP assumes that all losses are due to congestion; thus, loss detection also causes the sending rate to be reduced to a minimum and then increase gradually in order to probe the network's capacity [1].

Since wired links are extremely reliable, congestion is indeed the main cause of loss there. Congestion control requires end-to-end corrective actions; thus, it is sensible to combine it with loss recovery at the transport layer, simplifying the link layer and allowing applications that do not require absolute reliability to avoid it by using U DP. With wireless links in the picture, however, frequent noncongestion losses also occur, due to either wireless bit errors or communication pauses during handoffs. These cause the rate-limiting congestion control mechanisms of TCP to be triggered repeatedly even for relatively low error rates, reducing throughput over the bottleneck wireless link [2]. End-to-end recovery also increases delay, a significant problem for interactive applications.

Performance measurements of a $900 \mathrm{M} \mathrm{Hz}$ WLAN using U D P with 1400-byte packets over an $85 \mathrm{ft}$ distance showed an 


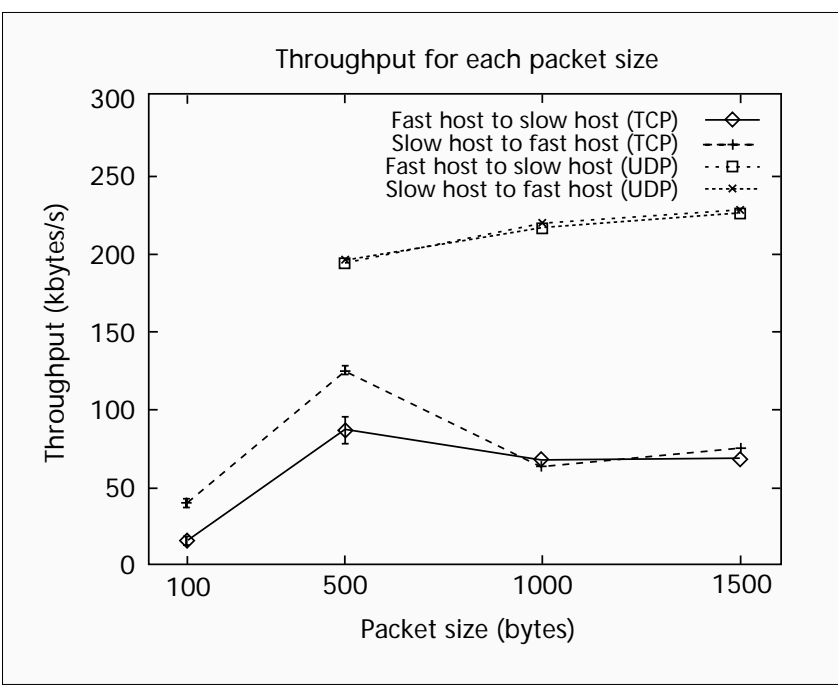

Figure 1. UDP and TCP throughput (ISA/ISA).

average error rate of 1.55 percent with clustered losses [3]. TCP generally achieves lower throughputs than UDP, not only due to its additional overhead, but also because reverse traffic (acknowledgments) must share broadcast WLA N s with forward (data) traffic. D elay and loss in broadcast media may also increase due to collisions. M easurements of TCP performance over a single-hop path (a WLA N link with 2 percent packet loss) showed that TCP throughput dropped to only 47 percent of its value in the absence of losses [4]. CT links on the North A merican code-division multiple access (CDM A) system exhibit 1-2 percent error rates over its 172-bit frames [5]. TCP/IP datagrams must be segmented into multiple such frames, causing the IP datagram error rate to increase dramatically and TCP performance to drop accordingly. CT systems use interleaving techniques to randomize frame losses and avoid degraded voice quality. R andom frame losses cause more I P datagrams to be lost than clustered losses, while interleaving also increases delay.

W hen multiple wireless links are included in a path, for example, when two wireless hosts communicate over the Internet, errors accumulate, further reducing throughput. W idearea paths are more sensitive to losses since larger transmission windows must be maintained to fully utilize them, and end-toend recovery is slower there. $\mathrm{H}$ andoffs in cellular systems are another problem since TCP can timeout during communications pauses. A $n$ emerging concept is hierarchical cellular systems. These combine different technologies (satellites, CT, WLANs) to support heterogeneous overlapping cells. R oaming between dissimilar systems requires vertical handoffs, in addition to horizontal handoffs within a system. Vertical handoffs change long-term end-to-end path characteristics.

\section{Case Study: A 2.4 GHz DSSS Wireless LAN}

We have performed a detailed study of the L ucent $2.4 \mathrm{G} \mathrm{Hz}$ $W$ aveL $A N$, a $2 \mathrm{M} \mathrm{b} / \mathrm{S} W L A N$ system that presents the same interface as an Ethernet LAN. We provide here a summary of our observations, while more details can be found in [6]. O ur measurements extend previous work in many ways: we used hosts with varying processing power and different types of interfaces (ISA and PCM CIA); we studied bidirectional (TCP) in addition to unidirectional (U DP) communications to evaluate the impact of contention and collisions between data and acknowledgments; and we used Linux instead of a Berkeley Software D istribution (BSD) derivative. We tested communication with single-hop (WLAN) paths over which we had full control. We used a stock Linux kernel with drivers modified to report more detailed statistics.
Both ISA and PCM CIA interfaces, despite differences in their $E$ thernet controllers and radio modules, implement the same collision avoidance medium access control (M A C) scheme and are fully compatible. This replaces the collision detection MAC scheme of wired $E$ thernets because collision detection is costly in terms of bandwidth for radio links. A fter sensing an idle medium, the transmitter waits for a random number of slots, and only if the medium is still silent does it transmit, to avoid excessive collisions. We used the ttcp benchmark which sends a number of packets using either UDP or TCP, modified to report loss statistics along with throughput. Each test consisted of sending 10,000 packets using either UDP or TCP, with different payloads $(100,500$, 1000 , and 1500 bytes). We repeated each test five times in both directions between each pair of hosts. We also used tcpdump to collect detailed packet traces.

We present here results from the baseline scenarios where the two communicating hosts are placed next to each other. The first scenario uses two ISA hosts; the second mixes one ISA and one PCM CIA host. F or the ISA -to-ISA case, one of the hosts is considerably faster, causing data transfers toward the slower host to experience losses of one out of every roughly 1000 transmitted packets, depending on packet size. Figure 1 plots TCP and U DP throughput (mean, minimum, and maximum across test repetitions) against packet size. U DP throughput peaks at $225 \mathrm{~kb} / \mathrm{s}(1.8 \mathrm{M} \mathrm{b} / \mathrm{s})$, higher than previously reported, due to increased host processing power. We do not show results for 100-byte packet U D P tests because the system dropped large bursts of those packets without sending them, due to protocol stack buffering limitations. TCP did not suffer from this problem due to its flow control.

The most interesting part is TCP performance with 1000 and 1500-byte packets where throughput drops to only about 30 percent of the corresponding U D P results. The cause can be seen in Fig. 2, which shows the mean, minimum, and maximum number of data and acknowledgment packets sent and received, with the faster host as the sender. The gaps between sent and received curves for both data and acknowledgments indicate losses that grow with packet size. These gaps are of the same magnitude, and are due to collisions undetected by the M A C layer. The packet traces show that the duplicate acknowledgments returned by the receiver after such a loss can also be lost to collisions. If the sender does not receive three duplicate acknowledgments, however, it does not detect

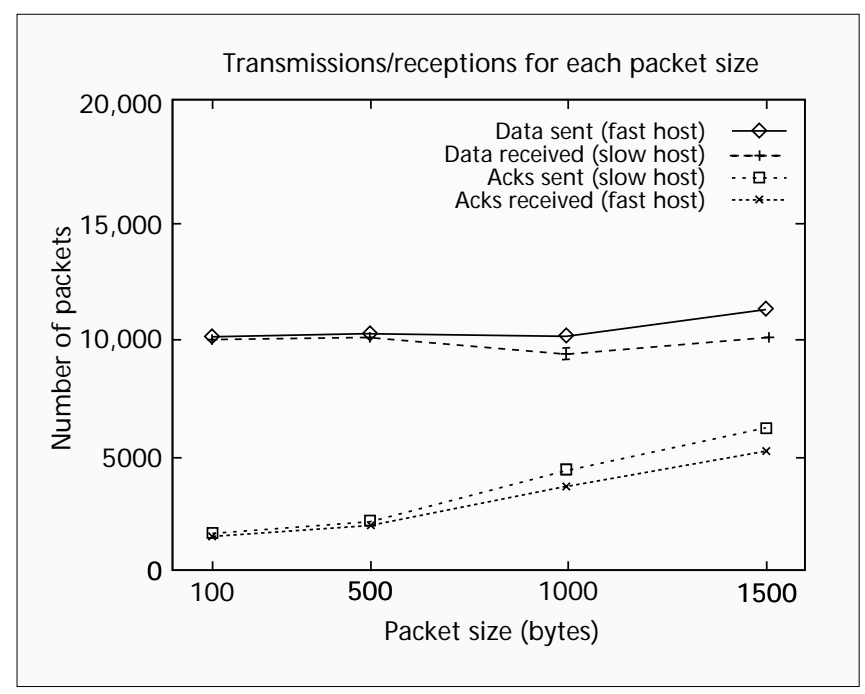

Figure 2. TCP data and acknowledgments (ISA/ISA). 
a loss. A similar problem occurs when the sender exhausts its transmission window before enough data packets are sent to trigger enough duplicate acknowledgments. In both cases, the sender must stall until a timeout occurs, which takes at least 200 ms in Linux and 500 ms in many B SD -derived systems, leaving the link idle. Timeouts also lead to more drastic reductions in TCP's transmission rate than loss detection via duplicate acknowledgments [1].

In the ISA-to-PCM CIA case, the ISA host is considerably faster, again causing losses when sending toward the slower PCMCIA host. The PCMCIA host occasionally drops packets without sending them due to the single transmit buffer of PCM CIA cards (ISA cards have multiple buffers), which is easier to overrun. Figure 3 shows TCP and U DP throughput in both directions. TCP throughput is lower in the PCM CIAto-ISA direction due to its less aggressive timing [3] and slower (single buffer) hardware implementation. In this scenario there are no excessive collisions due to the different timing of the two interfaces, leading to excellent TCP throughput. Interestingly, UDP is slower than TCP in the PCM CIA -to-ISA direction, despite TCP overhead and retransmissions. Packet traces indicate that occasionally the PCM CIA sender pauses for more than $1 \mathrm{~s}$, exactly when packets are lost. Since, unlike TCP, UDP does not provide flow control, sender buffer overruns may occur, causing interface resets. The resulting pauses cause peak U DP throughput to be only $160 \mathrm{~kb} / \mathrm{s}(1.28 \mathrm{M} \mathrm{b} / \mathrm{s})$ in this direction.

To summarize, asymmetries between processors and interfaces affect performance. Fast senders overrun slow receivers, causing packet losses, while PCM CIA cards lag behind ISA ones in transmission throughput. They are also prone to transmit buffer overruns and transmission pauses. The asymmetry between ISA and PCM CIA cards helps avoid the large number of undetected collisions that plague TCP performance in the ISA -to-ISA case. The impact of collisions on TCP is magnified by the loss of duplicate acknowledgments, which leads to timeout-initiated recovery. These shortcomings could be solved by M A C layer acknowledgments and retransmissions, thus enhancing TCP performance. UDP, however, does not face collision problems, and interactive applications using it may prefer transmitting new data instead. The effects of timeout delays point out the importance of timer granularity on TCP. A s long as adequate filtering is performed to avoid TCP instability, more accurate timers speed up recovery.

\section{Link Layer vs. Transport Layer Enhancements}

Since TCP is the most popular transport protocol on the Internet, and its problems with wireless links are due to its own assumptions, modifying TC $P$ has been a popular approach. The key is avoiding congestion control measures for noncongestion losses. For mobility-related losses, if signaling from lower layers is available, recovery may be initiated without a timeout [7]. However, for more frequent losses due to wireless errors, end-to-end recovery is too slow. O ne way to speed it up is to split TCP connections where wired and wireless links interface. $O$ ne instance of TCP executes over the wired part, while another instance of TCP or another protocol executes over the wireless part [8], with software agents connecting the two. This allows wireless losses to be handled locally. Splitting end-to-end connections in this manner may violate transport layer semantics. Complex software agents are required to synchronize each transport connection. A II TCP-specific solutions are furthermore inappropriate for other protocols, and since they operate at a high level they cannot exploit linkspecific optimizations.

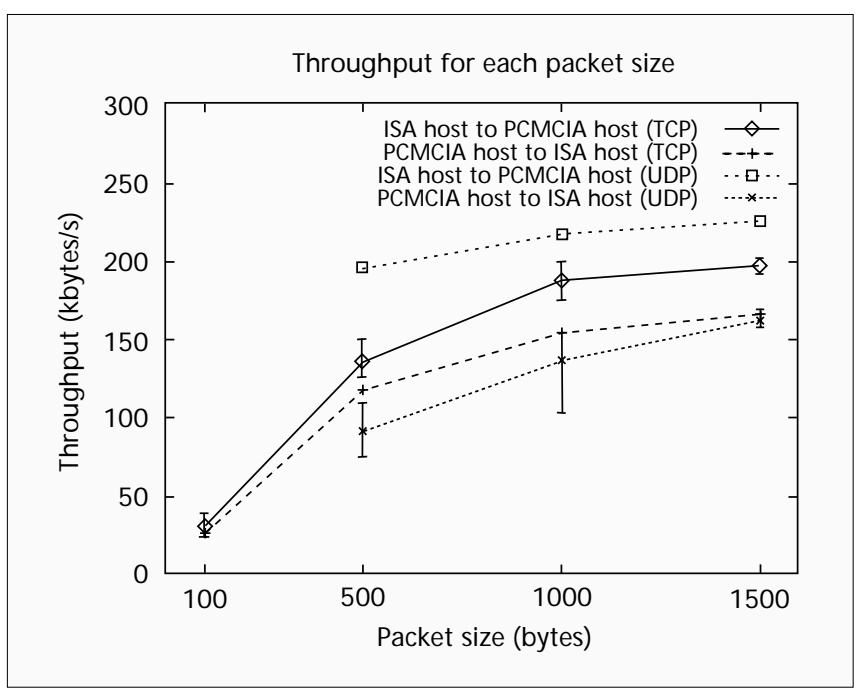

Figure 3. UDP and TCP throughput (ISA/PCMCIA).

The alternative to transport layer modifications is to improve the service offered on the link by providing error recovery at the link layer. Traditional link layers use retransmissions to provide full recovery, at the expense of variable delays. These may cause TCP to timeout in the process and trigger end-to-end loss recovery anyway [9]. In order to avoid such adverse interactions limited recovery may be provided, leaving further recovery to higher layers [5]. A nother variation is to provide transport-protocol-specific optimizations at the link layer [10]. By exploiting transport layer information, the link layer can avoid adverse interactions and economize on control overhead. This coupling, however, limits the applicability of such solutions to specific higher layers [2]. Link layer schemes generally have the advantage of working at the local level, with intimate knowledge of the wireless link and low round-trip delays that allow fast recovery. They can be deployed locally, transparent to the rest of the Internet. A TCP study found link layer schemes to outperform transport layer ones over W LA N links [4]. For these reasons, we decided to focus our research on link layer enhancements.

\section{Performance of Link Layer Enhancements}

\section{Simulation Environment and Setup}

G iven the diversity of higher-layer requirements, it is questionable whether a single link layer scheme is suitable for all applications. We thus undertook a simulation study to evaluate the performance of various types of mechanisms for different applications. We used N etwork Simulator version 2 (ns-2) [11]. N s-2 is oriented toward Internet simulations, containing very good implementations of many TCP variants. I ts source code is freely available; thus, researchers may extend the system by adding or modifying objects. The simulator is implemented in $\mathrm{O}$ bject $\mathrm{TCl}$ and $\mathrm{C}++$, allowing objects to be prototyped in the 0 bject $\mathrm{Tcl}$ interpreter and then compiled in $\mathrm{C}++$ if desired. We fully exploited these capabilities, adding wireless error models, link layer schemes, and applications.

W e describe here performance measurements using CT wireless links with $14.4 \mathrm{~kb} / \mathrm{s}$ of bandwidth (as in G lobal System for M obile Communications, G SM ) and a $100 \mathrm{~ms}$ frame delay (as in North A merican CDM A). The links employ fixedsize 50-byte frames and suffer from independent frame losses 


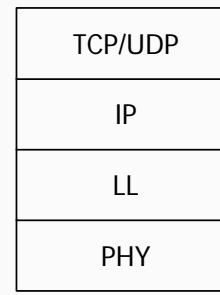

Wireless host

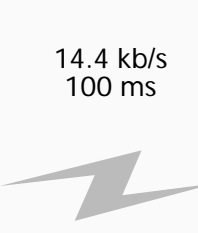

Base station

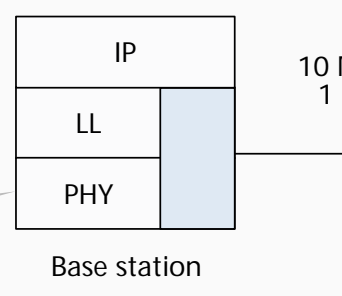

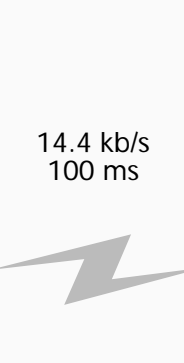

Figure 4. The simulation topology.

at rates of $1,2,5$, and 10 percent. The loss model and long delay are due to physical layer interleaving that aims to randomize frame losses [5]. Figure 4 depicts the two wireless link topology simulated which consists of two $\mathrm{CT}$ links joined by an $\mathrm{E}$ thernet ( $10 \mathrm{M} \mathrm{b} / \mathrm{s}, 1 \mathrm{~ms}$ ), with the communicating parties being two wireless users at either end of the path. We also simulated a simpler topology consisting of a single C T link and an $\mathrm{E}$ thernet, with the same parameters as above. In this topology the sender is at the wired end and the receiver at the wireless end of the path, simulating a wireless client receiving data from a wired server.

We tested both TCP and U DP applications with different requirements to examine the benefits of various link layer schemes for each type of application. For TCP, we simulated large file transfers using FTP over TCP R eno [1] with BSD style 500 ms granularity timers. In FTP tests the sender transmits $2 \mathrm{M}$ bytes of data to the receiver, with TCP handling flow, congestion, and error control on an end-to-end basis. W e ignored TCP/IP headers, assuming that header compression is performed over the slow wireless links. Thus, the total transfer consisted of 40,000 50-byte packets. We measured file transfer throughput, defined as the total size of the transfer (2 $M$ bytes), ignoring TCP and link layer overhead, divided by the total time taken to complete the transfer.

For UDP, we simulated one direction of an audio conference for $2000 \mathrm{~s}$. We used a speech model with silence suppression: when the speaker is active, data are transmitted at a constant bit rate (CBR) of $9.6 \mathrm{~kb} / \mathrm{s}$ (similar to N orth A merican CDM A); when the user is silent, no data are sent. Talking and silent periods have exponential durations, averaging $1 \mathrm{~s}$ and $1.35 \mathrm{~s}$, respectively [12]. The receiver buffers packets arriving at irregular intervals and plays them back isochronously, at a

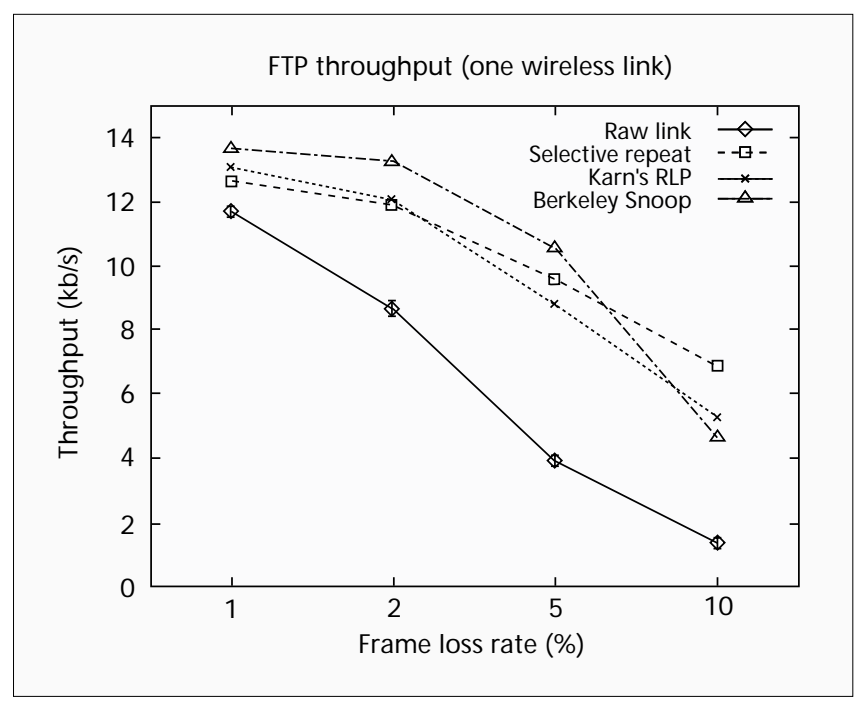

Figure 5. File transfer throughput, one wireless link. playback point determined by human perception. O ne metric for this application is the residual loss after link layer recovery. Since packets arriving after the playback point are unused, we are also interested in a delay bound for most packets. Thus, our delay metric was mean packet delay plus twice its standard deviation, which emphasizes the effect of variable delays. For all experiments we repeated each test 30 times with different random number generator seeds, calculating individual test metrics. We present here mean metric values among all tests, with error bars depicting the mean \pm 1 standard deviation.

For both TCP and UDP the raw link service is the performance baseline. Selective repeat is a window-based full recovery mechanism. The sender buffers outgoing frames, and retransmits those that are not acknowledged before a timeout. The receiver acknowledges and releases frames received in sequence. L ost frames are negatively acknowledged so that they can be retransmitted without a timeout. The scheme tested provides multiple negative acknowledgments per frame to reduce timeouts [13]. If a frame is lost repeatedly, the sender eventually exhausts its window and stalls; thus, delay is unbounded. A $n$ alternative is Karn's $R L P$, the radio link protocol of N orth A merican CDM A [5]. Negative acknowledgments are also used to trigger retransmissions, but if after a few (by default 3 ) retries the frame is not recovered, the receiver gives up, making the loss visible to higher layers. Limited recovery means that there is an upper bound on delay, and the sender never stalls. These schemes are suitable for TCP since they only release frames in sequence. Berkeley Snoop is a TCP-aware scheme [10]. It uses an agent at the base station (Fig. 4) that inspects TCP data and acknowledgments. Data sent to the wireless host are buffered, and if TCP acknowledgments indicate that a packet was lost, the packet is locally retransmitted. Snoop does not work for the reverse direction where TCP acknowledgments are returned from a remote host.

For the UDP conferencing application, delay is more important than full recovery. Forward error correction (FEC) schemes provide limited recovery but fixed delay bounds by adding redundancy in the transmitted stream to allow the receiver to reconstruct the original data despite losses. The XOR-based FEC scheme we tested transmits the original data frames unmodified, but every 8 frames a parity frame is constructed by XOR ing the preceding data frames. If a single data frame is lost, it can be recovered by XOR ing the remaining frames with the parity frame. FEC overhead is wasted when zero or more than one losses occur. This FEC scheme was selected for its simplicity. E ven though it is not among the most powerful, it exhibits the basic behavior of FEC schemes. We also tested UDP over Karn's R LP with one retransmission, providing a fixed delay bound. Losses in this scheme cause subsequent frames that are correctly received to wait until the missing frame is received or abandoned. This insequence delivery is critical for TCP, but useless for applications with playback buffers. Thus, we also tested an 


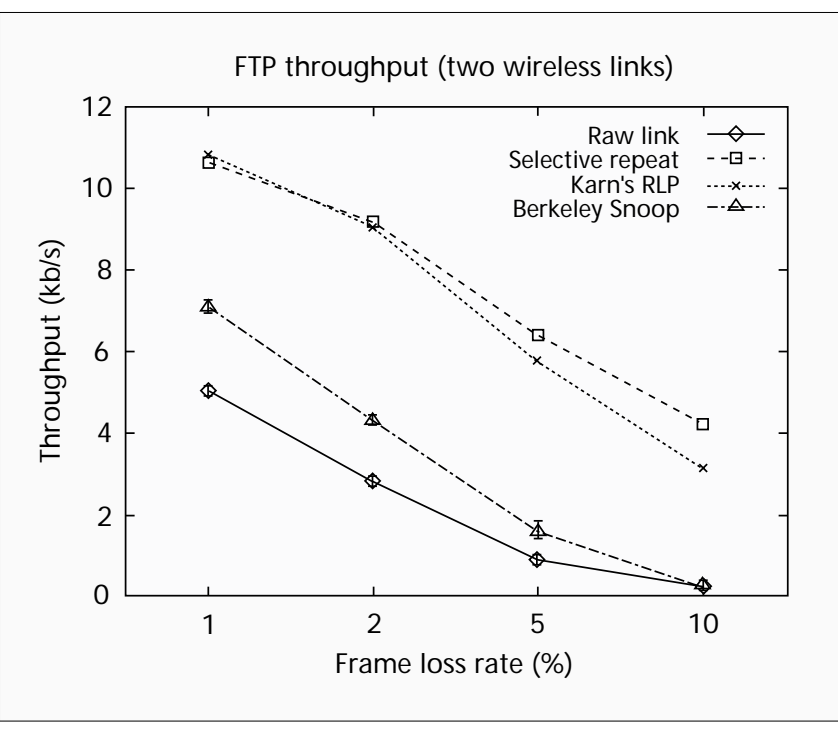

Figure 6. File transfer throughput, two wireless links.

out-of-sequence (O OS) R L P variant that reduces delay by releasing received frames immediately. When a path contains multiple wireless links, this prevents frames from being repeatedly delayed due to unrelated losses.

\section{File Transfer Performance over TCP}

Figure 5 shows file transfer throughput in the one wireless link topology (one E thernet and one wireless CT link). TCP data flow from the wired to the wireless host with acknowledgments travelling in the reverse direction. E ach curve depicts average test throughput for a particular link layer scheme under varying error rates. The raw link curve shows performance without any enhancements, pointing out that losses disproportionately affect TCP performance: a 5 percent loss rate causes TCP to achieve less than 30 percent of the maximum throughput. The best performer in this topology is Berkeley Snoop for all but the highest loss rate. This is the most economical scheme in terms of overhead because it exploits existing TCP headers to detect losses. Karn's R LP uses one byte of overhead per frame, while selective repeat uses two bytes per frame. A t higher error rates selective repeat is better, indicating that even though limited recovery avoids conflicting TCP and link layer retransmissions [9], it is better to recover without resorting to TCP. Since nearly all delay on this path is over the wireless link, the gains of link layer schemes are due not to local recovery but to more robust error control. At the highest loss rate Snoop loses ground because it mimics $T C P$ error recovery which is not very robust under harsh error conditions.

In the two-wireless-link topology, file transfer throughput, shown in Fig. 6, reveals a different picture. 0 ver the raw link TCP achieves about half of its throughput in the previous topology. Karn's R LP and selective repeat provide large performance improvements even under low loss rates, with selective repeat becoming more attractive under harsher error conditions. Berkeley Snoop, however, offers only minor improvements over the native service. The reason is its inability to improve performance in both directions over each wireless link. In this topology data cross the first wireless link in the wirelessto-base direction and the second in the base-to-wireless direction. Snoop improves only the latter; hence, losses in the former are visible to TCP. The same problem occurs in wireless-to-wired host transfers or with interactive applications like W eb browsing that exchange data in both directions. This problem cannot be avoided by placing an agent at the wireless host, since acknowledgments in this direction are returned much later by a remote host, hence negating the advantages of local recovery. The other link layer schemes do not face this problem because they use local acknowledgments for error detection.

\section{Conferencing Performance over UDP}

For the conferencing application the first metric of interest is residual loss, shown in Fig. 7 for the one wireless link topology. Instead of selective repeat and Berkeley Snoop, which are inappropriate for delay-sensitive traffic, we used XOR -based FEC and OOS RLP, with a one retransmission limit for both $R$ L P variants. O OS R LP was inappropriate for TCP because it did not deliver data in sequence. The raw link curve shows the native loss rate of the wireless link. The inefficiency of the FEC scheme is clear at the 10 percent loss rate: despite its 12.5 percent overhead ( 1 parity frame/8 data frames) it only reduces the error rate to 6 percent. $B$ oth $R L P$ variants keep loss rates below 2 percent in all cases, with identical performance throughout the error rate range, as expected. Loss rates for the two wireless link topology (not shown) are essentially twice those of Fig. 7. These results confirm that both $R L P$ schemes provide more efficient error recovery than XOR -based FEC.

Figure 8 shows the delay metric for the one wireless link scenario, with the raw link curve depicting the native delay. The worst performer is Karn's R LP: as loss rates grow, delay increases dramatically due to the large number of retransmissions that inflate delay variability. Lost packets also delay subsequently received ones due to in-sequence delivery. $O$ ut-of-sequence $R L P$, on the other hand, never delays received packets; hence, it is very close to XOR -based FEC, the best-performing scheme. $R L P$ recovery delay is determined by round-trip delay, while FEC delay is determined by block size (and one-way propagation delay).

W ith two wireless links the delay metrics, shown in Fig. 9, are similar: XOR -based FE C depicts the lowest delay among link layer enhancement schemes, closely followed by $00 \mathrm{~S}$ $R L P$, with K arn's $R L P$ exhibiting very large delays at higher error rates. While Karn's R LP increases its delay compared to the one wireless link topology by 90 percent, O O S R LP increases it by 70 percent; thus, the gap between them widens. This confirms that O OS delivery is more beneficial with multiple wireless links since packets are not repeatedly delayed due to unrelated losses. This is a very important point for multihop wireless networks.

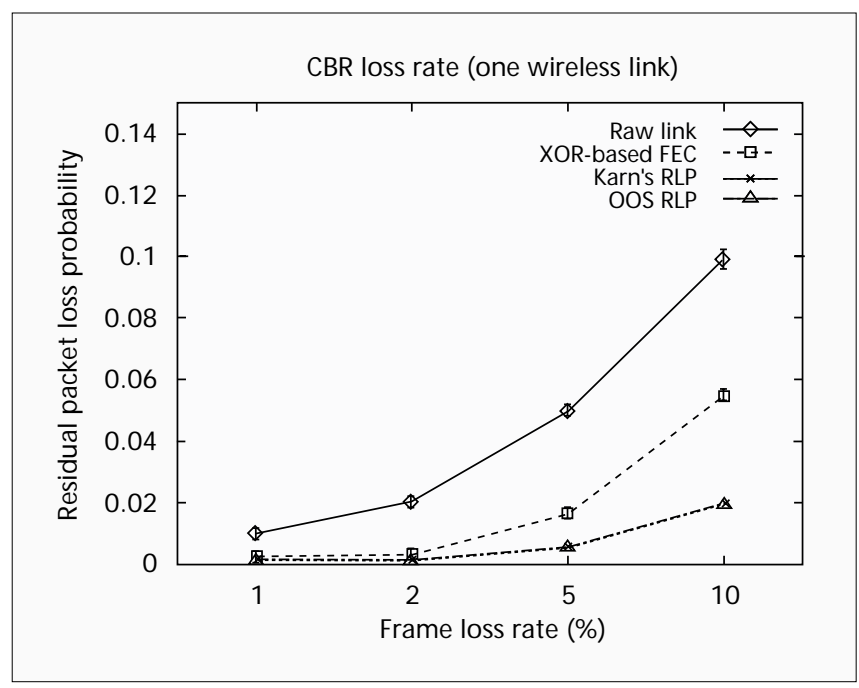

Figure 7. Conferencing loss, one wireless link. 


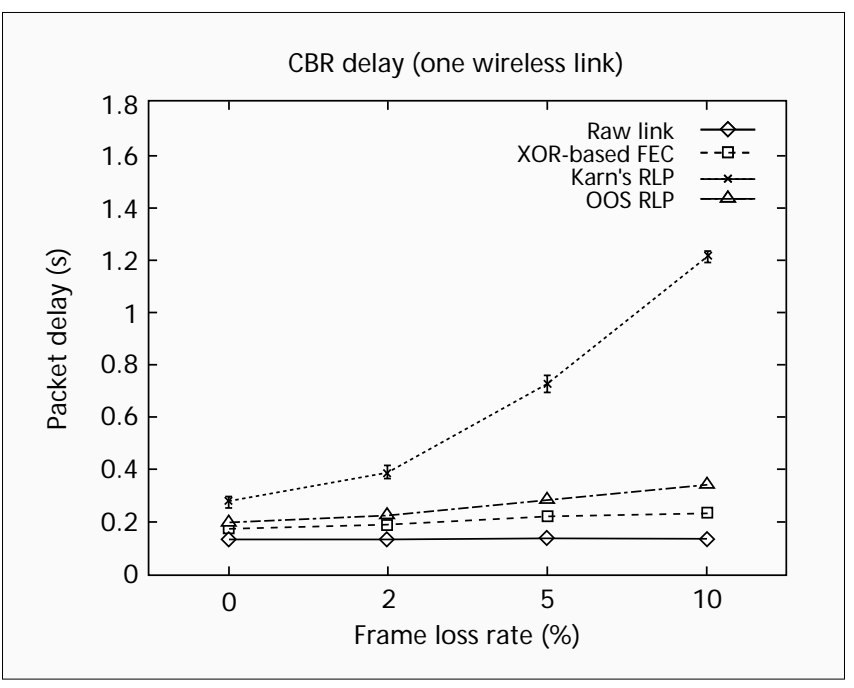

Figure 8. Conferencing delay (mean $+2 x$ standard deviation), one wireless link.

\section{The Multiservice Link Layer Approach}

\section{The Case for Multiple Link Layer Services}

O ur results show that link layer schemes can significantly improve Internet performance over wireless links. L ink layer mechanisms provide a local solution to a problem that is inherently dependent on and isolated to individual links. U nlike higher-layer schemes, their scope is limited to the endpoints of the wireless link. L ocal schemes can be optimized for each type of link since hardware details are accessible at the link layer. E rror recovery overhead is minimized, since no retransmitted or parity frames travel beyond the wireless link. D elays are also minimized since awareness of link characteristics can be used to set tight timeout intervals.

H owever, our results also indicate that different applications prefer fundamentally different enhancement mechanisms. TCP-based applications are relatively insensitive to delay but very sensitive to losses, favoring full recovery schemes. R ealtime U DP-based applications are relatively error-tolerant but very delay-sensitive, favoring limited recovery schemes. Future applications may exhibit other unforeseen requirements. As the Internet evolves toward providing multiple Q oS points, as in the differentiated services architecture [14], the limitations of link layer schemes providing a single service will become more apparent. In order to provide multiple Q oS points and support applications with diverse requirements, we are thus proposing a multiservice link layer architecture offering multiple simultaneous services over each wireless link. The architecture is easy to extend with new services catering to future requirements. Providing multiple services at the link layer is probably the only way to locally handle wireless impairments in a protocol-independent manner.

\section{Internal Design}

In order to provide multiple simultaneous services at the link layer we need to handle a number of design issues. Services must be isolated from each other to simplify their implementation; at the same time they must share link resources in an orderly manner. The multiservice aspect must be transparent to the existing Internet, which assumes that only a single service is offered, but visible to and easy to integrate with future Q oS-based higher layers and applications. Figure 10 depicts the internal design of our multiservice link layer architecture. A single entry point is provided for compatibili-
CBR delay (two wireless links)

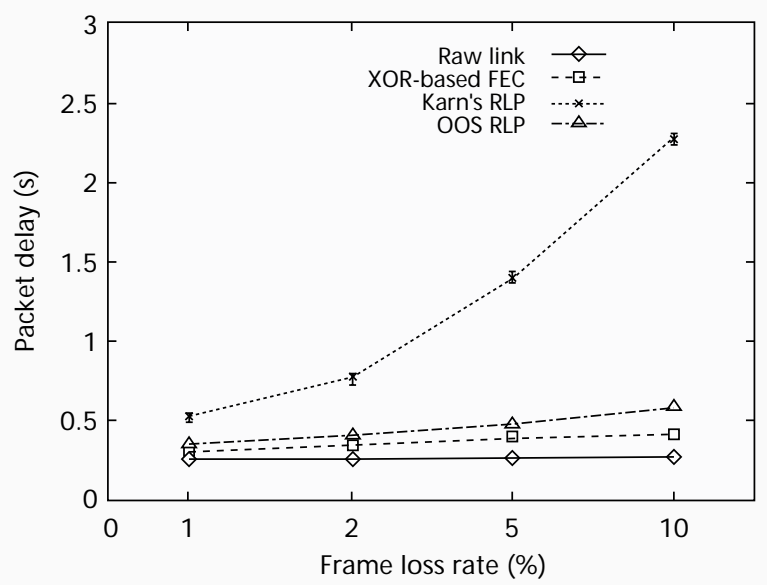

Figure 9. Conferencing delay (mean + 2 x standard deviation), two wireless links.

ty with IP, with an internal packet classifier distributing incoming packets to appropriate services. The classifier examines a number of IP and TCP/U D P header fields in order to map higher-layer requirements to available services. Q oS-based higher layers can explicitly set up these mappings, or the scheduler may heuristically map packets to services to ease integration with the existing Internet. Packets that are not explicitly matched to customized services are handled by the raw link service.

$\mathrm{H}$ igher layers expect the link layer to allocate transmission bandwidth to packets in the proportions presented to it by the network layer. This is especially important for higher layers that provide Q OS guarantees based on packet scheduling. Preserving bandwidth allocations is trivial in traditional link layers, but more complicated with multiple services. If a service provides error recovery, whether using retransmissions or data encoding, the overhead it generates inflates its data stream relative to other services. In order to allow each service to use any mechanism desired but prevent it from adversely interacting with the rest, we measure service bandwidth allocations at

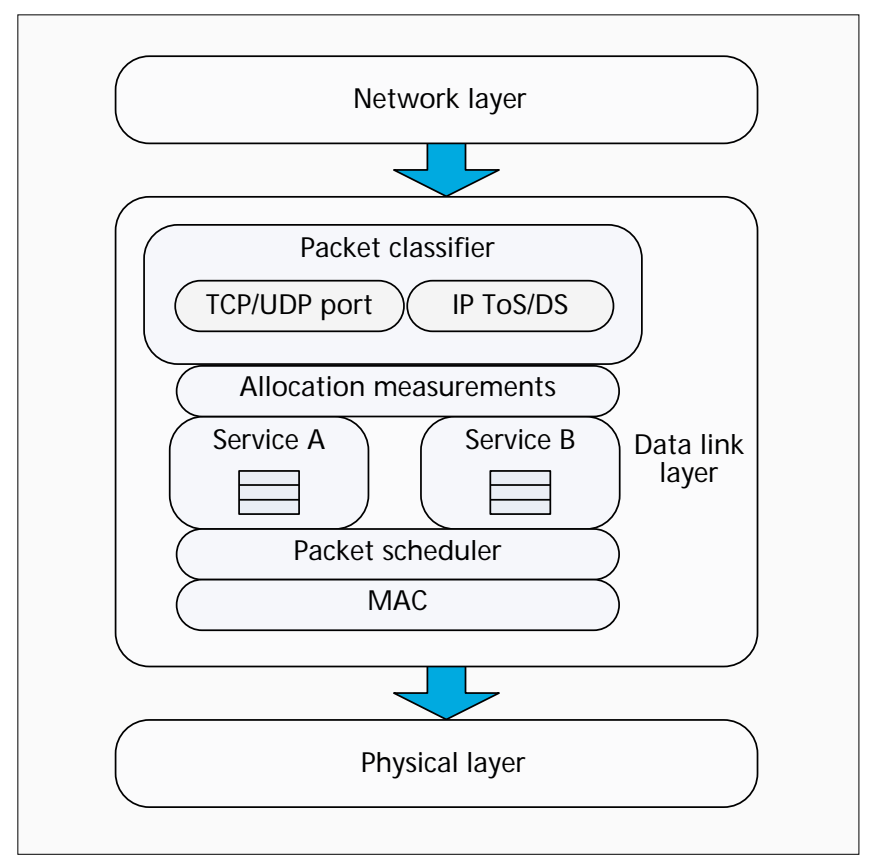

Figure 10. M ultiservice link layer architecture. 


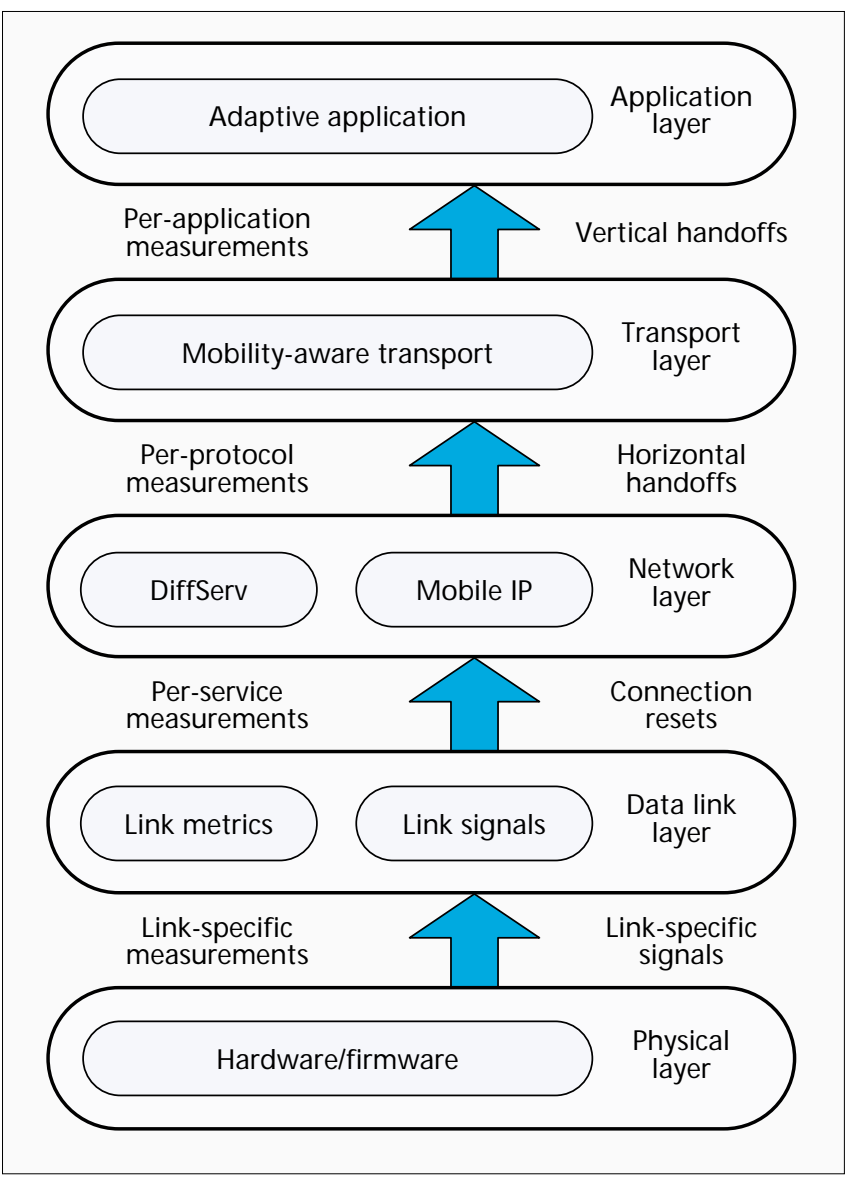

Figure 11. F eedback propagation through the protocol stack.

the entry to the link layer and enforce the same allocations over the physical link. The allocation measurement module tracks the fraction of data allocated to each service by the classifier over a time interval, before services inflate their data streams. These shares are enforced by the packet scheduler over the next time interval.

W e decided to use a self-clocked fair queuing (SCF Q ) scheduler because it is very efficient to operate [15]. SCFQ schedulers strictly enforce the desired bandwidth allocations when the link is loaded. When some services are inactive, their bandwidth is shared among the active ones; hence, the link is never left idle when there are data to send. N ote that the goal of the link layer scheduler is not end-to-end QoS provision but the preservation of higher-layer scheduling decisions despite arbitrary service overheads. This design isolates services from each other, since each can operate as if it was a conventional link layer protocol with a single peer at the other end of the link, using any recovery mechanisms desired and taking advantage of any possible link-specific optimizations.

Incoming packets are passed to services by the classifier using a generic call. Services pass packets to the scheduler using per-service queues. The scheduler decides which packet to send next based on the measured bandwidth allocations, labels it with a service number, and hands it to the M A C layer for transmission. A the receiver, incoming packets from the M A C layer are demultiplexed based on their service label and passed to the appropriate service, which may eventually pass them to the network layer. Hence, the classifier and scheduler are independent of the actual services implemented, and therefore reusable. A dding or removing services only requires modifying the mapping tables of the classifier, making the scheme very easy to extend.

\section{External Interface}

For existing Internet protocols, the interface presented by our multiservice link layer scheme is identical to that of traditional link layers, with single entry and exit points. A pplication-specific enhancements take place transparently to the rest of the stack. Q oS-aware higher layers, on the other hand, need information about the services offered to select the best one for each type of traffic. Since each wireless link has different characteristics, rather than attempting to standardize the offered services across the Internet, it is preferable to allow dynamic discovery of their characteristics. Thus, each service in our scheme exports dynamically updated device-independent metrics of its recent performance. Figure 11 depicts this upward flow of information through the protocol stack.

The physical layer may provide hardware-specific performance feedback to the link layer, which combines it with its own measurements. E ach service separately tracks and reports its performance metrics (i.e., residual loss, delay, and throughput). Throughput is normalized to reflect service performance as if it were using the link exclusively, to make it independent of past bandwidth allocations. M etrics are expressed in link-independent units such as bits and seconds. Besides making dynamic service selection possible, these metrics allow higher layers to estimate end-to-end path performance before and during a session [16]. The metrics may be used directly or modified by intermediate layers, for example, to account for their own error recovery mechanisms.

The link layer cannot handle mobility problems due to handoffs, because one of the two endpoints changes after a handoff. Due to its intimate knowledge of the underlying hardware, it can, however, help higher layers deal with mobility. N otifications, in the form of upcalls to higher layers, can inform interested parties of events, such as disconnections and resets, which may indicate the beginning and end of handoffs. These events are detected by link layer state changes or physical layer signals and are propagated in a standardized manner through the protocol stack. They can be filtered by higher layers into authoritative mobility notifications ( $\mathrm{Fig}$. 11), thus enabling protocols and applications to adapt their mechanisms accordingly. For example, a disconnect notification and a connect notification from two different link endpoints indicate to the network layer that a handoff occurred.

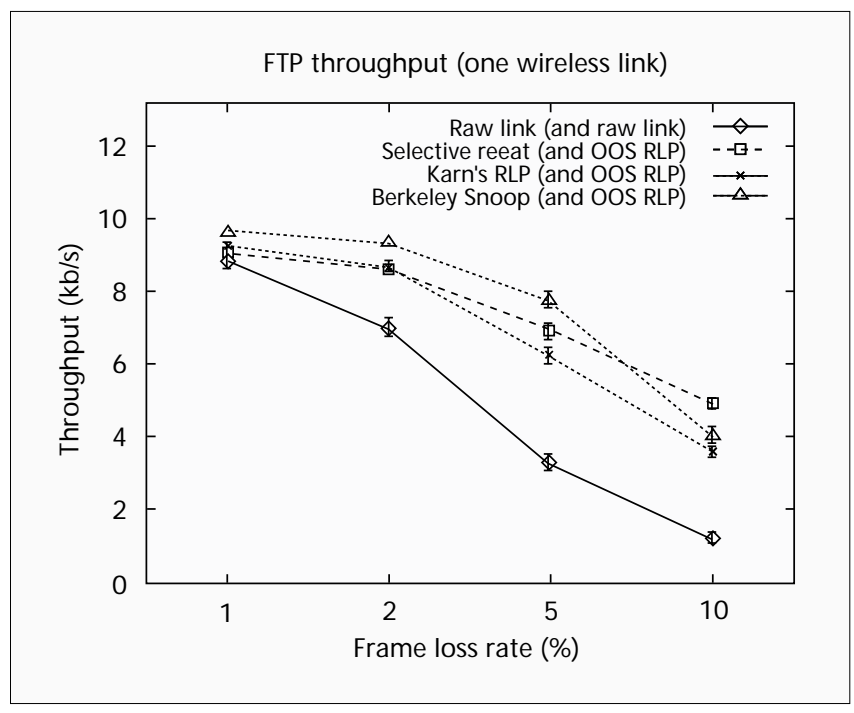

Figure 12. File transfer throughput, one wireless link. 


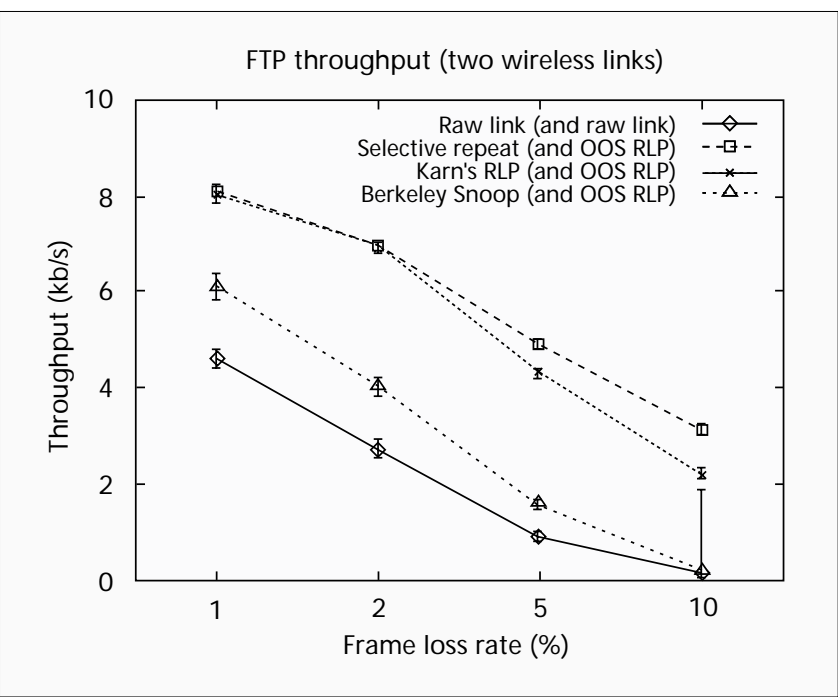

Figure 13. File transfer throughput, two wireless links.

\section{Integration with the Internet}

For our link layer architecture to succeed, it must seamlessly integrate with both existing and future (Q OS-aware) Internet protocols. E ach service provided employs a mix of mechanisms optimized for the underlying medium, possibly adapting to current link conditions. Services are meant to satisfy generic requirements rather than fit the specific details of TCP or UDP applications. In the absence of any explicit instructions, application requirements may be inferred by examining I $P$ and TCP/U D P headers. The classifier may use fields such as protocol type, TCP/U D P port number for applications using wellknown ports, or the IP Type of Service (TOS) field to heuristically map incoming traffic to link layer services. U nrecognized traffic is passed to the raw link (or other default) service. $\mathrm{N}$ ew services may be added as needed to expand the range of supported traffic classes or handle future requirements.

F or Q oS-based higher layers, our link layer enhances the offered service without interfering with higher-layer decisions. When packet scheduling is used to control bandwidth sharing during congestion, as in Class-Based Queuing (CBQ ) [17], our link layer preserves such allocations. The overhead introduced by each service does not violate higher-layer scheduling since our scheduler allocates bandwidth based on the incoming data stream before it is inflated by each service. $\mathrm{H}$ igher layers aware of the multiple services offered may create enhanced end-to-end services using R SV P for setup and negotiations [18]. The performance metrics exported by each service can be used to select and characterize services. When differentiated services are used to provide various Q OS grades to different traffic classes, the same IP DS field may be used to map packets to services at both levels [14].

Link layer feedback can be further refined as it flows upwards through the protocol stack, as shown in Fig. 11. $M$ obile IP can rapidly detect mobility using link layer notifications, in turn issuing separate notifications for horizontal and vertical handoffs. Horizontal handoffs can be dealt with at the transport layer, for example, by freezing TCP timers until the handoff completes. Vertical handoffs change long-term path behavior, so they are propagated to applications. A video application, for example, could adapt by switching to a different resolution. The services available on the new link can be discovered by looking at the exported metrics. End-to-end services can then be renegotiated by signaling protocols such as R SV P. H igher-layer packet scheduling schemes such as CB Q may also use the exported metrics to predict the outcome of their scheduling decisions.
Deployment of the proposed multiservice link layer architecture on the existing Internet is eased by its emulation of a single service link layer. Both endpoints of each wireless link are usually under the control of a single administrative entity; thus, both sides can upgrade their link layer software at the same time, transparent to the rest of the Internet. G eneric services, such as those discussed earlier, may be provided at first to efficiently support all TCP and some U DP applications, with further services added as future needs arise. This is attractive to equipment vendors that could deploy our scheme to gain a competitive advantage on the market.

\section{Multiservice Link Layer Performance}

To evaluate our architecture we added a multiservice link layer object to the ns-2 simulator (as discussed before). O ur implementation includes a heuristic classifier that maps packets to services based on their protocol (TCP or UDP) and an SCFQ scheduler, both capable of handling an arbitrary number of services. Services are implemented using any of the link layer enhancement schemes described previously. We wanted to determine whether our approach is capable of simultaneously enhancing the performance of multiple applications by the same factor as when executing each application in isolation over the same link layer mechanism, adjusted for the reduced bandwidth due to link sharing. We thus simulated simultaneous file transfer over TCP and conferencing over UDP using the same CT links, topologies, and application parameters as before. Both applications start simultaneously over the same path, and the simulation ends when FTP completes sending $2 \mathrm{M}$ bytes of data.

E ach wireless endpoint employs a multiservice link layer module with one TCP and one UDP service. We statically allocated $9.6 \mathrm{~kb} / \mathrm{s}$ of bandwidth (out of $14.4 \mathrm{~kb} / \mathrm{s}$ ) to the UDP service, satisfying the peak $C B R$ requirements of the conferencing application. Since the U DP sender is active 42.5 percent of the time, the average bandwidth available to TCP is $10.3 \mathrm{~kb} / \mathrm{s}$. To establish a performance baseline we used the raw link service for both UDP and TCP. E nhanced performance was provided by OOS RLP for UDP, coupled with selective repeat, Karn's R LP, or Berkeley Snoop for TCP. We show average results from 30 test repetitions.

Figure 12 shows file transfer throughput in multiservice tests using the one wireless link topology, while Fig. 13 shows the same metric with the two wireless link topology. The ser-

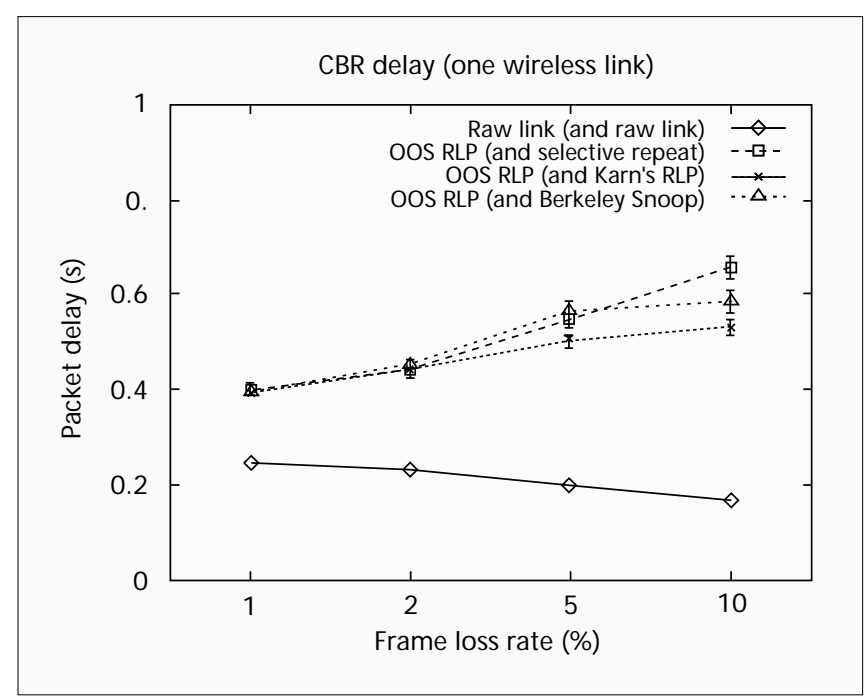

- Figure 14. Conferencing delay (mean $+2 x$ standard deviation), one wireless link. 


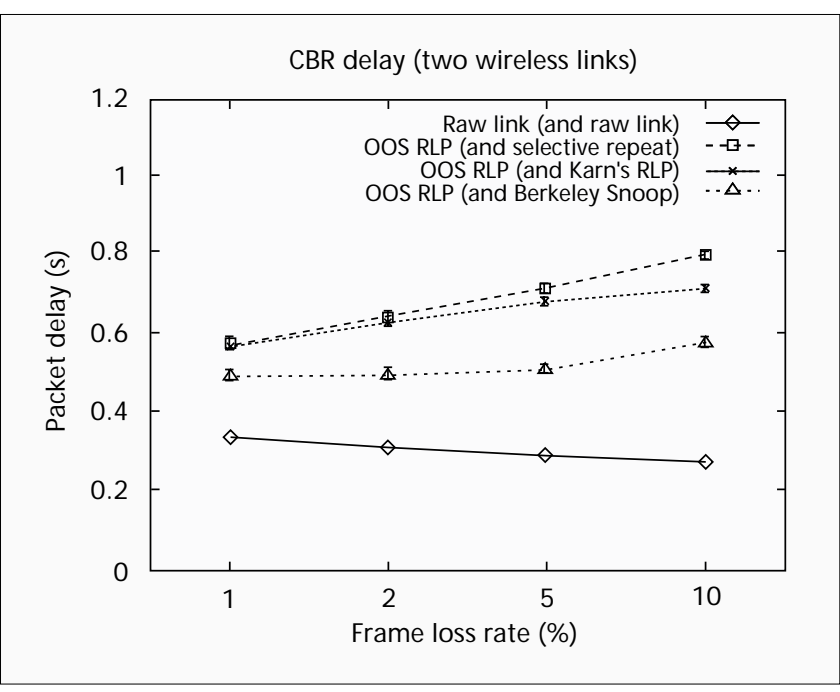

Figure 15. Conferencing delay (mean +2 xstandard deviation), two wireless links.

vice used for UDP is shown in parentheses. The shape and relative position of all throughput curves are nearly identical to those shown in Figs. 5 and 6. While performance is reduced for all schemes due to competing UDP traffic, the factor of improvement over the raw link service is virtually the same as in single-application tests using the same link layer mechanism. Thus, TCP performance over multiservice links is improved in exactly the same manner as for a single service.

F or the UDP service, residual loss results (not shown) are exactly the same as in single service tests ( $\mathrm{Fig.} \mathrm{7).} \mathrm{Figure} 14$ shows the delay metric for conferencing in multiservice tests with one wireless link, while Fig. 15 shows the same metric with two wireless links. The service used for TCP is shown in parentheses. D elay is inflated compared to the raw link scenario due to OOS RLP recovery delay (Figs. 8 and 9) and contention with TCP traffic. When TCP uses the raw link service, its performance drops with higher error rates; hence, $U D P$ delay also drops. When enhanced T C P services are used, TCP performance improves; hence, UDP delay increases. Note, however, that delay increases in proportion to the improvement in TCP performance and not to error recovery overhead. Thus, the scheduler manages to protect UDP delay performance from TCP service overhead.

\section{Conclusion}

We outlined the problems faced by Internet protocols over wireless links and argued in favor of link layer enhancements. We presented simulations showing that diverse applications are best served by fundamentally different link layer schemes. W e thus proposed a multiservice link layer architecture that simultaneously improves the performance of multiple types of applications by combining arbitrary link layer mechanisms over a single link. Our link layer exports information about its services to allow enhanced end-to-end services to be created. Services are isolated from each other to ease programming.
O ur architecture can be transparently integrated with the existing Internet. 0 ur simulations show that our approach offers virtually the same improvements to each stream sharing the link as those of single application tests, the best we could have hoped for while preserving fairness.

\section{References}

[1] W. Stevens, "TCP Slow Start, Congestion Avoidance, Fast Retransmit and Fast Recovery Algorithms," RFC 2001, Jan. 1997.

[2] G. Xylomenos and G. C. Polyzos, "Internet Protocol Performance over Networks with Wireless Links," IEEE Network, vol. 13, no. 5, July/Aug. 1999.

[3] G. T. Nguyen et al., "A Trace-Based Approach for Modeling Wireless Channel Behavior," Proc. Winter Sim. Conf., 1996.

[4] H. Balakrishnan et al., "A Comparison of Mechanisms for Improving TCP Performance over Wireless Links," Proc. ACM SIGCOMM '96, Aug. 1996, pp. 256-67.

[5] P. Karn, "The Qualcomm CDMA Digital Cellular System," Proc. USENIX Mobile and Location-Independent Comp. Symp., Aug. 1993, pp. 35-39.

[6] G. Xylomenos and G. C. Polyzos, "TCP and UDP Performance over a Wireless LAN," Proc. IEEE INFOCOM ‘99, Mar. 1999, pp. 439-46.

[7] R. Càceres and L. Iftode, "Improving the Performance of Reliable Transport Protocols in Mobile Computing Environments," IEEE JSAC, vol. 13, no. 5, June 1995, pp. 850-57.

[8] B. R. Badrinath et al., "Handling Mobile Clients: A Case for Indirect Interaction," Proc. 4th Wksp. Workstation Op. Sys., Oct. 1993, pp. 91-97.

[9] A. DeSimone, M. C. Chuah, and O.C. Yue, "Throughput Performance of Transport-Layer Protocols over Wireless LANs," Proc. IEEE GLOBECOM ‘93, Dec. 1993, pp. 542-49.

[10] H. Balakrishnan, S. Seshan, and R. H. Katz, "Improving Reliable Transport and Handoff Performance in Cellular Wireless Networks," Wireless Networks, vol. 1, no. 4, 1995, pp. 469-81.

[11] “UCB/LBNL/VINT Network Simulator - ns (Version 2)," available at http://www-mash.cs.berkeley.edu/ns

[12] S. Nanda, D. J. Goodman, and U. Timor, "Performance of PRMA: A Packet Voice Protocol for Cellular Systems," IEEE Trans. Vehic. Tech., vol. 40, no. 3, Aug. 1991, pp. 584-98.

[13] P. T. Brady, "Evaluation of Multireject, Selective Reject, and Other Protocol Enhancements," IEEE Trans. Commun., vol. 35, no. 6, June 1987, pp. 659-66.

[14] S. Blake et al., "An architecture for Differentiated Services," RFC 2475 Dec. 1998.

[15] S. Golestani, "A Self-Clocked Fair Queuing Scheme for Broadband Applications," Proc. IEEE INFOCOM ‘94, June 1994, pp. 636-46.

[16] S. Shenker and L. Breslau, "Two Issues in Reservation Establishment," Proc. ACM SIGCOMM '95, Oct. 1995, pp. 14-26.

[17] S. Floyd and V. Jacobson, "Link-Sharing and Resource Management Models for Packet Networks," IEEE/ACM Trans. Networking, vol. 3, no. 4, Aug. 1995, pp. 365-86.

[18] L. Zhang et al., "RSVP: A New Resource Reservation Protocol," IEEE Network, vol. 7, no. 5, Sept. 1993, pp. 8-18.

\section{Biographies}

GEORGE XYLOMENOS [S'96] (xgeorge@cs.ucsd.edu) holds a diploma in informatics (1993) from Athens University of Economics, Greece, and an M.S. (1996) and a Ph.D. (1999), both in computer science, from the Department of Computer Science and Engineering at the University of California, San Diego. His research focuses on improving the performance of Internet applications over wireless links, providing QoS over wireless and mobile networks, and reconciling the notions of local and end-to-end QoS.

GEORGE C. Polyzos [S'80-M'88] (polyzos@cs.ucsd.edu) received a Dipl. E.E. from National Technical University, Athens, Greece, and an M.A.Sc. in E.E. and a Ph.D. in computer science from the University of Toronto, Canada. He joined the faculty of the Department of Computer Science and Engineering at the University of California, San Diego in September 1988. He is a member of the UCSD Center for Wireless Communications, Senior Fellow of the San Diego Supercomputer Center, and co-director of the Computer Systems Laboratory. His research interests include communications networks and multimedia systems design and performance evaluation. 